\title{
Es liegt nicht alles am Sozialismus - über Ost-West-Unterschiede und ihre Ursprünge
}

\begin{abstract}
Auch zum 30. Jubiläum der Deutschen Einheit ist noch viel von Ost-West-Unterschieden die Rede. Im Vordergrund stehen hierbei neben rein ökonomischen Differenzen auch Unterschiede in soziokulturellen Einstellungen und Verhaltensweisen. Dass diese Diskussionen auch nach so langer Zeit noch geführt werden, erscheint erstaunlich und wirft die Frage nach der Herkunft dieser anhaltenden Differenzen auf. Typischerweise wird diese Frage mit der Erfahrung verschiedener politischer Systeme beantwortet, die unterschiedliche gesellschaftliche Prägungen zur Folge hatten. Folglich entwickelte sich auch in den Sozialwissenschaften ein großes Interesse an der deutschen Teilung 1949 in die Deutsche Demokratische Republik und die Bundesrepublik Deutschland sowie an der Wiedervereinigung 1990. Die Bevölkerung in Ost- und Westdeutschland unterschied sich jedoch bereits vor der Teilung. Die heute verbleibenden Unterschiede müssen dennoch nicht als unveränderlich hingenommen werden.
\end{abstract}

\begin{abstract}
Für Sozialwissenschaftler:innen ist es von großer Bedeutung, die Auswirkungen verschiedener gesellschaftlicher Interventionen zu untersuchen. Dazu zählt auch, zu verstehen, wie sich Kommunismus, Demokratie und Autokratie auf das Leben der Menschen auswirken. Es überrascht daher nicht, dass es eine breite Literatur gibt, die
(C) Der/die Autor:in(nen) 2021. Open Access: Dieser Artikel wird unter der Creative Commons Namensnennung 4.0 International Lizenz veröf- fentlicht (creativecommons.org/licenses/by/4.0/deed.de).

Open Access wird durch die ZBW - Leibniz-Informationszentrum Wirtschaft gefördert.
\end{abstract}

Prof. Dr. Sascha O. Becker ist Xiaokai Yang Chair of Business and Economics an der Monash University, Melbourne/Australien und Professor an der University of Warwick in Coventry/Großbritannien.

Dr. Lukas Mergele ist wissenschaftlicher Mitarbeiter am ifo Zentrum für Bildungsökonomik des ifo Instituts in München.

Prof. Dr. Ludger Wößmann leitet das ifo Zentrum für Bildungsökonomik am Münchener ifo Institut und lehrt Volkswirtschaft an der Ludwig-MaximiliansUniversität München. untersucht, wie politische Systeme die Wirtschaft und die Präferenzen der Bevölkerung nachhaltig beeinflussen. Ein besonderer Schwerpunkt liegt dabei auf den unterschiedlichen Vermächtnissen von kapitalistischen und sozialistischen Systemen (Simpser et al., 2018 für einen Überblick). Jedoch ist es unmöglich, politische Systeme zu randomisieren, was sozialwissenschaftliche Untersuchungen erschwert und die Suche nach alternativen Forschungsmethoden erfordert.

Ost-West-Unterschiede nach der deutschen Teilung und Wiedervereinigung

Vor allem die deutsche Teilung und Wiedervereinigung hat das Interesse vieler Sozialwissenschaftler:innen geweckt, da sie einer experimentellen Situation recht nahe zu kommen scheint. Nach dem Zweiten Weltkrieg wurden zwei zuvor vereinte Teile desselben Landes zwei gegensätzlichen politischen Regimen zugeordnet, dem kapitalistischen Westen und dem kommunistischen Osten. Die Wiedervereinigung 1990 führte die beiden Teile unter demselben politischen System wieder zusammen. ${ }^{1}$

In Bezug auf die gesamtwirtschaftliche Lage unterschied sich das Pro-Kopf-Einkommen vor dem Zweiten Weltkrieg zwischen Ost- und Westdeutschland nicht wesentlich (Alesina und Fuchs-Schündeln, 2007). Zum Zeitpunkt des Zusammenbruchs der Deutschen Demokratischen Republik (DDR) betrug das Bruttoinlandsprodukt pro

1 Teile dieses Beitrags basieren auf Becker, Mergele und Wößmann (2020b). 
Kopf in Ostdeutschland dagegen weniger als die Hälfte des westdeutschen. Nach der Wiedervereinigung lag die Arbeitsproduktivität in Ostdeutschland bei einem Drittel des Westniveaus, womit der Osten zwischen der Produktivität Mexikos und Chiles lag. Das kommunistische System war wirtschaftlich gescheitert.

Angesichts der markanten Unterschiede zwischen den politischen und wirtschaftlichen Systemen in West- und Ostdeutschland wurde in der Literatur vielfach argumentiert, dass die kommunistische Erfahrung nachhaltige Auswirkungen auf die Bevölkerung im Osten hatte, einschließlich ihrer wirtschaftlichen Folgen, politischen Einstellungen, kulturellen Besonderheiten und Geschlechterrollen (z. B. Alesina und Fuchs-Schündeln, 2007; Campa und Serafinelli, 2019; Laudenbach et al., 2019; GoldfaynFrank und Wohlfart, 2020; Lippmann et al., 2020).

In einer neuen Studie (Becker, Mergele und Wößmann, 2020a) werfen wir einen neuen Blick auf diesen Aspekt der deutschen Geschichte. Waren Ost- und Westdeutschland vor dem Zweiten Weltkrieg tatsächlich ähnlich? Haben der Krieg und die nachfolgenden Besatzungsmächte die beiden Landesteile in gleicher Weise beeinflusst? Wie steht es mit der Migration zwischen Ost und West von 1945 bis zum Bau der Berliner Mauer 1961? Und was bedeutet das alles für unser Verständnis der Auswirkungen des Kommunismus?

Die DDR ist schon zu erkennen, bevor sie überhaupt existierte

Der Verlauf der Grenze zwischen der DDR und der Bundesrepublik Deutschland ist nicht das zufällige Ergebnis davon, wo amerikanische, britische und sowjetische Panzer am Ende des Zweiten Weltkriegs 1945 zum Stehen kamen. Die drei alliierten Streitkräfte hatten sich in Erwartung einer Niederlage Nazi-Deutschlands bereits 1944 auf eine Aufteilung des Nachkriegs-Deutschlands in sowjetische und westliche Besatzungszonen geeinigt, die den Grenzen der Teilgebiete des Deutschen Reichs und der preußischen Provinzen vor dem Zweiten Weltkrieg folgte (mit einigen sehr geringfügigen Ausnahmen zwecks besserer geografischer Anbindung). Dementsprechend trennte die deutschdeutsche Grenze die Bevölkerungen bereits existierender Regionen mit unterschiedlicher Geschichte und Kultur.

Da die Grenze zwischen bestehenden administrativen Einheiten verlief, können wir Kreisdaten aus der Zeit vor dem Zweiten Weltkrieg verwenden, um zu untersuchen, ob sich West und Ost in relevanten Dimensionen unterschieden haben. Eine erste Dimension ist die Bedeutung der Arbeiterklasse, die von den kommunistischen Ländern stark betont wird. Wenn wir die Daten aus der Zeit

\section{Abbildung 1}

Der Anteil der Arbeiterklasse im Jahr 1925: Ost-West-Unterschiede vor Existenz der DDR

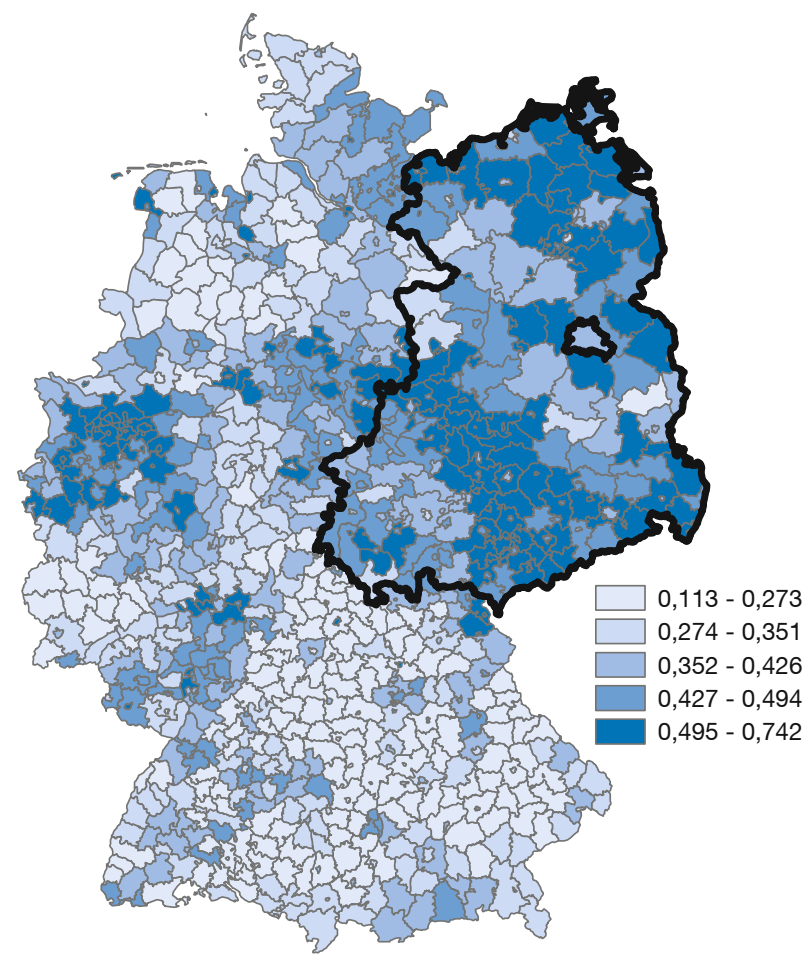

Anmerkungen: Farben beziehen sich auf Quintile. Fehlende Daten werden durch nächste Nachbarn imputiert.

Quelle: Eigene Berechnungen basierend auf Falter and Hänisch (1990); Becker, Mergele und Wößmann (2020a).

vor dem Zweiten Weltkrieg untersuchen, sehen wir jedoch, dass die spätere DDR bereits 1925 einen wesentlich höheren Anteil der Arbeiterklasse hatte (vgl. Abbildung 1), lange bevor das Gebiet kommunistisch wurde. Der Unterschied beträgt 12 Prozentpunkte oder ein Drittel des westlichen Niveaus. Tatsächlich steigt der Anteil der Arbeiterklasse in mehreren Regionen rund um die spätere innerdeutsche Grenze recht abrupt an: Der Unterschied ist deutlich erkennbar, wenn man sich nur auf die Kreise im Umkreis von 100 Kilometern um die spätere Grenze konzentriert oder sogar auf die Kreise, die direkt an die spätere Grenze angrenzen.

Ost-West-Unterschiede vor dem Zweiten Weltkrieg sind in vielen weiteren Dimensionen erkennbar. Abbildung 2 zeigt Vergleiche in weiteren wirtschaftlichen Strukturen, die für den Beginn des Kommunismus relevant sind. Unter anderem war der Anteil der Beschäftigten in Industrie und Handwerk im Osten deutlich höher, während der Anteil der Selbstständigen deutlich geringer war (Fritsch und Wyrwich, 2014). 


\section{Abbildung 2}

Ost-West-Unterschiede vor dem Zweiten Weltkrieg

Wirtschaft

Anteil der Arbeiterklasse (1925)

Beschäftigungsanteil im Verarbeitenden Gewerbe (1925)

Anteil der Selbstständigen (1925)

Politische Präferenzen

Stimmanteil der kommunistischen Partei (1924)

Stimmanteil der linken Partei (1924)

Kultur

Geschlechterrollen

Anteil Protestant:innen (1925)

Kirchenbesuch (1900-1910)

Weibliche Erwerbsbeteiligung (1925)

Außereheliche Geburtenrate (1937)

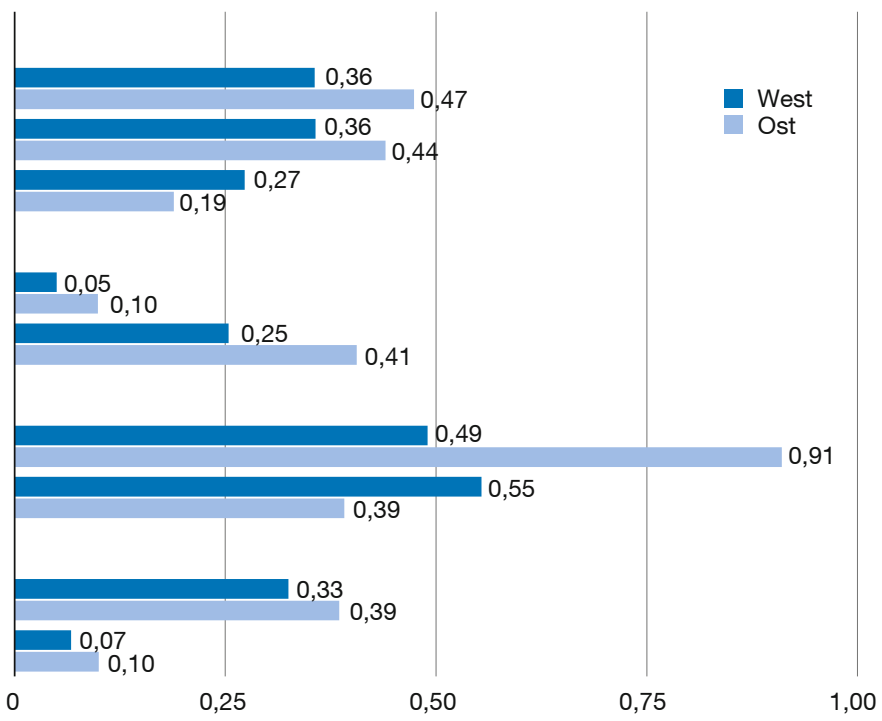

Quelle: eigene Darstellung auf der Grundlage von Becker, Mergele und Wößmann (2020a).

Interessanterweise unterschieden sich auch die politischen Präferenzen bereits vor dem Zweiten Weltkrieg. Bei der Reichstagswahl im Dezember 1924 war der Stimmenanteil der Kommunistischen Partei Deutschlands im Osten doppelt so hoch wie im Westen. Betrachtet man die linken Parteien im weiteren Sinne (insbesondere unter Hinzunahme der Sozialdemokratischen Partei Deutschlands), so lag der Stimmenanteil der linken Parteien im Osten um 15 Prozentpunkte höher.

Der Kommunismus wird oft mit der Verdrängung von Religion assoziiert. Ostdeutschland wies jedoch bereits zu Beginn des 20. Jahrhunderts einen deutlich geringeren Kirchenbesuch (in der evangelischen Kirche) auf (Hölscher, 2001). Zudem setzte sich der Westen in etwa gleichen Teilen aus Protestant:innen und Katholik:innen zusammen, während der Osten überwiegend (91\%) protestantisch war (Becker und Wößmann, 2009).

Schließlich legte die sozialistische DDR großen Wert darauf, Frauen in Arbeit zu bringen. Doch schon vor dem Zweiten Weltkrieg war die Erwerbsbeteiligung von Frauen im Osten höher (Wyrwich, 2019). Zudem unterschieden sich Ost und West in der Häufigkeit außerehelicher Geburten (Klüsener und Goldstein, 2016). In dem Maße, in dem einige dieser zuvor bestehenden Unterschiede auch während der kommunistischen Periode fortbestanden, könnten sie eine wesentliche Quelle der Heteroge- nität zwischen Ost- und Westdeutschen nach der Wiedervereinigung sein.

Der Zweite Weltkrieg und die Besatzungsmächte wirkten sich in Ost und West unterschiedlich aus

Ost- und Westdeutschland waren auch vom Zweiten Weltkrieg und den Besatzungsmächten unterschiedlich betroffen. Anhand von Daten der deutschen Volkszählung, die im Oktober 1946 in allen vier Besatzungszonen gemeinsam durchgeführt wurde, zeigen wir, dass der Quotient von Männern zu Frauen in der Sowjetzone wesentlich geringer war. Bei der letzten Volkszählung vor dem Zweiten Weltkrieg 1939 gab es keine derartigen Unterschiede. Der stärkere Rückgang des Geschlechterverhältnisses in der Sowjetzone könnte eine größere kriegsbedingte männliche Todesrate widerspiegeln, möglicherweise aber auch Geschlechterunterschiede in der sehr frühen OstWest-Migration. Was auch immer die Ursache, diese Differenz könnte unabhängig vom Kommunismus zu Unterschieden etwa in der Erwerbsbeteiligung von Frauen oder den Geschlechterrollen beigetragen haben.

Der Osten erlitt auch größere Verluste durch die Demontage von Investitionsgütern durch die Besatzungsmacht und die Zahlung von Reparationen an die Besatzungsmacht im Zeitraum 1945 bis 1949. Dies verschaffte der DDR eine schlechtere Ausgangslage (Sleifer, 2006). 
Ein Fünftel der Bevölkerung verließ den Osten vor dem Bau der Berliner Mauer

Die Besatzungszonen wurden 1945 eingerichtet, die DDR wurde 1949 gegründet. Dennoch war es möglich, wenn auch zunehmend schwierig, zwischen den beiden Teilen Deutschlands zu migrieren, bis 1961 die Berliner Mauer gebaut wurde. Tatsächlich wanderte bis 1961 etwa jeder fünfte Einwohner Ostdeutschlands in den Westen aus. Es gibt zwar keine Daten, die einen Vergleich der Eigenschaften dieser Auswandernden mit denen, die im Osten zurückblieben, erlauben. Wir zeigen jedoch, dass sich die Personen, die von Ost nach West zogen, von den einheimischen Westdeutschen durch einen größeren Anteil Angestellter, Selbstständiger und höher gebildeter Personen unterschieden. Vermutlich waren sie auch weniger empfänglich für die kommunistische Doktrin (auch Bauernschuster et al., 2012).

Darüber hinaus wird manchmal übersehen, dass auch etwa eine halbe Mio. Menschen vor 1961 aus dem Westen in den Osten eingewandert sind. Die DDR-Propaganda beschreibt sie als "nicht einverstanden mit dem kapitalistischen System“, wobei in vielen Fällen auch familiäre Gründe eine Rolle gespielt haben dürften. Wir zeigen, dass sechs der 19 Mitglieder des Politbüros der frühen DDR (1949 bis 1961) in dem Gebiet der späteren Bundesrepublik geboren wurden, darunter auch Erich Honecker. Zusammengenommen deuten die Befunde darauf hin, dass es eine große selektive Migration und eine Sortierung nach politischen Präferenzen gab.

Interpretation von Auswirkungen des Kommunismus nur mit Vorsicht

Lässt sich die deutsche Situation angesichts dieser Erkenntnisse über bereits bestehende Ost-West-Unterschiede, unterschiedliche Betroffenheit durch den Zweiten Weltkrieg sowie selektive Ost-West-Migration überhaupt nutzen, um die Auswirkungen des Kommunismus zu untersuchen? Wir denken, dass die Antwort „ja“ lautet, da sie einige einzigartige Vorteile bietet. Wir betonen jedoch, dass jede Forschungsfrage die skizzierten Herausforderungen berücksichtigen muss. Die überzeugendsten Belege für die Auswirkungen politischer Systeme ergeben sich vermutlich aus der Konvergenz einiger wirtschaftlicher Verhaltensweisen, politischer Präferenzen und des Vertrauens in den Staat zwischen Ost und West nach der Wiedervereinigung. Auch scheint die Erfahrung, im kommunistischen System gelebt zu haben, die Konsummuster dauerhaft verändert zu haben. Darüber hinaus dürfte das kommunistische System die Geschlechterrollen etwa in Bezug auf die weibliche Erwerbsbeteiligung geprägt haben, wobei diese auch eine starke bereits zuvor bestehende Komponente aufweisen.

\section{Implikationen für die öffentliche Debatte}

Mit Blick auf die öffentliche Debatte bedeuten weit in die Geschichte zurückgehende Unterschiede nicht, dass diese für immer zementiert sind und als gegeben akzeptiert werden müssten. Immerhin lässt sich seit der Wiedervereinigung eine beachtliche Konvergenz von Einstellungen und Verhaltensweisen feststellen (Rainer et al., 2018). Dass dieser Prozess nach einer Generation allerdings nicht zu einer vollständigen Angleichung geführt hat, wird durch den historischen Rückblick umso verständlicher. Eine offene Forschungsfrage ist jedoch, warum in manchen Lebensbereichen die Konvergenz sehr stark ist, in anderen hingegen die Persistenz der Unterschiede (noch) überwiegt.

Wie Unterschiede überwunden werden können, zeigen beispielhaft drei aktuelle Forschungsergebnisse:

1. Gemeinsame Medien können eine Öffentlichkeit schaffen, durch die eine Annäherung möglich ist. Slavtchev und Wyrwich (2017) vergleichen ostdeutsche Gebiete, die in der DDR die Empfangsmöglichkeit von Westfernsehen hatten, mit Regionen, denen dies aufgrund geografischer Gegebenheiten nicht möglich war. Ihre Analyse zeigt, dass in Gebieten mit Zugang zu westlichen Medien nach der Wiedervereinigung mehr Unternehmensgründungen erfolgten und gründungsfreundlichere Einstellungen vorherrschten.

2. Kontakt- und Begegnungsmöglichkeiten fördern gemeinsame Einstellungen. Stegmann (2019) analysiert hierzu eine Maßnahme der DDR-Regierung, die es der in grenznahen Kreisen lebenden Bevölkerung einfacher machte, private Besuche aus dem Westen zu empfangen. Der Vergleich mit Regionen, die von diesen Erleichterungen nicht profitierten, zeigt, dass die einfacheren Austauschmöglichkeiten demokratische Präferenzen förderten und die Proteste gegen das DDR-Regime im Revolutionsjahr verstärkten.

3. Auch innerdeutsche Migration kann die Angleichung von Einstellungen und Verhaltensweisen fördern. So finden Schmitz und Weinhardt (2019), dass sich die Erwerbsbeteiligung westdeutscher Frauen in Regionen mit hoher Zuwanderung aus Ostdeutschland seit der Wiedervereinigung besonders stark ausgeweitet hat. Die Deutsche Einheit hat somit auch Westdeutschland nachhaltig verändert.

Der Blick in die Geschichte der Ost-West-Unterschiede kann uns also lehren, mit einiger Gelassenheit auf heutige Unterschiede zu blicken. Regionale Differenzen an sich bedeuten noch kein Unheil. Solange ausreichend Gemeinsamkeiten vorhanden sind, können sie sogar bereichern. 


\section{Literatur}

Alesina, A. und N. Fuchs-Schündeln (2007), Goodbye Lenin (or not?): The effect of communism on people, American Economic Review, 97(4), 1507-1528.

Bauernschuster, S., O. Falck, R. Gold und S. Heblich (2012), The shadows of the socialist past: Lack of self-reliance hinders entrepreneurship, European Journal of Political Economy, 28(4), 485-497.

Becker, S. O., L. Mergele und L. Wößmann (2020a), The separation and reunification of Germany: Rethinking a natural experiment interpretation of the enduring effects of communism, Journal of Economic Perspectives, 34(2), 143-171.

Becker, S. O., L. Mergele und L. Wößmann (2020b), Die deutsche Teilung und Wiedervereinigung und die „Auswirkungen“ des Kommunismus, ifo Schnelldienst, 73(5), 48-51.

Becker, S. O. und L. Wößmann (2009), Was Weber wrong? A human capital theory of Protestant economic history, Quarterly Journal of Economics, 124(2), 531-596.

Campa, P. und M. Serafinelli (2019), Politico-economic regimes and attitudes: Female workers under state socialism, Review of Economics and Statistics, 101(2), 233-248.

Fritsch, M. und M. Wyrwich (2014), The long persistence of regional levels of entrepreneurship: Germany, 1925-2005, Regional Studies, 4(6), 955-973.

Goldfayn-Frank, O. und J. Wohlfart (2020), Expectation formation in a new environment: Evidence from the German reunification, Journal of Monetary Economics, 115, 301-320.

Hölscher, L. (2001), Datenatlas zur religiösen Geographie im protestantischen Deutschland: Von der Mitte des 19. Jahrhunderts bis zum Zweiten Weltkrieg, Walter de Gruyter.
Klüsener, S. und J. R. Goldstein (2016), A long-standing demographic East-West divide in Germany, Population, Space and Place, 22(1), 5-22.

Laudenbach, C., U. Malmendier, A. Niessen-Ruenzi (2019), The longlasting effects of experiencing communism on attitudes towards financial markets, Working Paper.

Lippmann, Q., A. Georgieff und C. Senik (2020), Undoing gender with institutions: Lessons from the German division and reunification, ECOnomic Journal, 130(629), 1445-1470.

Rainer, H., C. Albrecht, S. Bauernschuster, A. Fichtl, T. Hener und J. Ragnitz (2018), Deutschland 2017 - Studie zu den Einstellungen und Verhaltensweisen der Bürgerinnen und Bürger im vereinigten Deutschland, ifo Forschungsberichte, 96.

Simpser, A., D. Slater und J. Wittenberg (2018), Dead but not gone: Contemporary legacies of communism, imperialism, and authoritarianism, Annual Review of Political Science, 21(1), 419-439.

Schmitz, S. und F. Weinhardt (2019), Immigration and the Evolution of Local Cultural Norms, IZA Discussion Paper, 12509.

Slavtchev, V. und M. Wyrwich (2017), TV and entrepreneurship, IWH Discussion Papers, 17/2017, Halle Institute for Economic Research (IWH).

Sleifer, J. (2006), Planning Ahead and Falling Behind: The East German Economy in Comparison with West Germany 1936-2002, Jahrbuch für Wirtschaftsgeschichte, 8, Akademie Verlag.

Stegmann, A. (2019), When East meets West: Interpersonal Contact and the demand for democracy, Working Paper.

Wyrwich, M. (2019), Historical and current spatial differences in female labour force participation: Evidence from Germany, Papers in Regional Science, 98(1), 211-239.

Title: It Is Not All Because of Socialism - On East-West Differences and Their Origins

Abstract: Even on the 30th anniversary of German unity, there is still a lot of talk about East-West differences. Typically, these differences are attributed to different political systems, which have created different impressions. As a result of the division of Germany in 1949 into the GDR and the Federal Republic as well as the reunification in 1990, a lively interest in the social sciences has developed as they offer a unique framework for analysing the effects of communism. Never before has such an unexpected introduction and abolition of a communist regime occured on the territory of a previously and subsequently united country. This paper looks into the history of East-West differences and argues that the division and reunification of Germany are nonetheless not a simple "experiment" from which one can directly deduce the effects of communism. The paper finds that the populations of East and West Germany already differed before the division, that the GDR and FRG were unequally affected by the Second World War, and that selective East-West migration took place during the division. Nevertheless, the remaining East-West differences are not necessarily permanent. 